\section{D) Check for updates}

Cite this: Nanoscale, 2021, 13, 15481

\title{
Retraction: Polymer nanodots of graphitic carbon nitride as effective fluorescent probes for the detection of $\mathrm{Fe}^{3+}$ and $\mathrm{Cu}^{2+}$ ions
}

\author{
Shouwei Zhang, ${ }^{\mathrm{a}, \mathrm{b}}$ Jiaxing $\mathrm{Li}^{\mathrm{c}}{ }^{\mathrm{C}}$ Meiyi Zeng, ${ }^{\mathrm{b}}$ Jinzhang $\mathrm{Xu},{ }^{\mathrm{b}}$ Xiangke Wang ${ }^{\star \mathrm{a}, \mathrm{c}}$ and \\ Wenping $\mathrm{Hu}^{* \mathrm{~d}}$
}

DOI: $10.1039 / \mathrm{d} 1 \mathrm{nr} 90195 \mathrm{~h}$

Retraction of 'Polymer nanodots of graphitic carbon nitride as effective fluorescent probes for the detection of $\mathrm{Fe}^{3+}$ and $\mathrm{Cu}^{2+}$ ions' by Shouwei Zhang et al., Nanoscale, 2014, 6, 4157-4162, DOI: 10.1039/

rsc.li/nanoscale C3NR06744K.

The Royal Society of Chemistry, with the agreement of the authors, hereby wholly retract this Nanoscale article due to concerns with the reliability of the data in the published article. Repeating fragments can be observed in the TEM images in Fig. 1B and $\mathrm{C}$, indicating that these images have been manipulated. The first author initially requested to retract this article because he admitted that Photoshop technology was used in the preparation of Fig. 1. The authors subsequently requested to correct the article based on updated experimental data. The authors apologize for any inconvenience to readers.

Signed: Shouwei Zhang, Jiaxing Li, Meiyi Zeng, Jinzhang Xu, Xiangke Wang and Wenping $\mathrm{Hu}$

Date: 11th August 2021

Retraction endorsed by Michaela Mühlberg, Executive Editor, Nanoscale.

\footnotetext{
${ }^{a}$ School for Radiological and Interdisciplinary Sciences, Soochow University, Suzhou 215123, PR China. E-mail: xkwang@ipp.ac.cn, xkwang@suda.edu.cn; Fax: +86-551-65591310; Tel: +86-551-65592788

${ }^{b}$ School of Materials Science and Engineering, Hefei University of Technology, Hefei 230031, PR China

${ }^{c}$ Key Laboratory of Novel Thin Film Solar Cells, Institute of Plasma Physics, Hefei 230031, PR China

${ }^{d}$ Beijing National Laboratory for Molecular Sciences, Key Laboratory of Organic Solids, Institute of Chemistry, Chinese Academy of Sciences, Beijing 100190, PR China. E-mail: huwp@iccas.ac.cn; Fax: +86-10-62527295; Tel: +86-10-82615030
} 